\title{
Real-World Safety and Effectiveness of Dimethyl Fumarate in Black or African American Patients with Multiple Sclerosis: 3-Year Results from ESTEEM
}

\author{
Mitzi J. Williams · Lilyana Amezcua • Annette Okai • Darin T. Okuda · \\ Stanley Cohan · Ray Su • Becky Parks · Jason P. Mendoza • \\ James B. Lewin · Cynthia C. Jones
}

Received: April 2, 2020 / Published online: May 29, 2020

(C) The Author(s) 2020

\section{ABSTRACT}

Introduction: Black or African American (black/ AA) patients with multiple sclerosis (MS) are reported to exhibit greater disease severity compared with non-black or non-AA patients. Whether differences exist in response to MS diseasemodifying therapies remains uncertain, as MS

Digital features To view digital features for this article go to https://doi.org/10.6084/m9.figshare.12205916.

B. Parks was an employee of Biogen at the time the research was conducted.

M. J. Williams

Joi Life Wellness MS Center, Atlanta, GA, USA

L. Amezcua

Keck School of Medicine, University of Southern California, Los Angeles, CA, USA

A. Okai

Multiple Sclerosis Treatment Center of Dallas, Dallas, TX, USA

D. T. Okuda

Neuroinnovation Program, UT Southwestern

Medical Center, Dallas, TX, USA

S. Cohan

Providence Multiple Sclerosis Center, Providence Brain and Spine Institute, Portland, OR, USA

R. Su · B. Parks · J. P. Mendoza · J. B. Lewin ·

C. C. Jones $(\bowtie)$

Biogen, Cambridge, MA, USA

e-mail: cynthia.jones@biogen.com clinical trials have included low numbers of nonwhite patients. We evaluated real-world safety and effectiveness of dimethyl fumarate (DMF) on MS disease activity in black/AA patients.

Methods: ESTEEM is an ongoing, 5-year, multinational, prospective study evaluating long-term safety and effectiveness of DMF in patients with MS. This interim analysis included patients newly prescribed DMF in routine practice at 394 sites globally.

Results: Overall, 4897 non-black/non-AA and 187 black/AA patients were analyzed; median (range) follow-up 18 (2-37) months. Unadjusted annualized relapse rates (ARRs) for 12 months before DMF initiation versus 36 months post DMF initiation, respectively, were: non-black/non-AA patients, 0.83 (95\% CI $0.80-0.85)$ versus 0.10 (95\% CI 0.09-0.10), 88\% lower ARR ( $P<0.0001)$; black/AA patients, 0.68 (95\% CI 0.58-0.80) versus 0.07 (95\% CI $0.05-0.10)$, 90\% lower ARR $(P<0.0001)$. In total, 35 (19\%) black/AA patients reported adverse events leading to treatment discontinuation; gastrointestinal disorders were most common (7\%), consistent with non-black/non-AA patients (8\%). Median lymphocyte counts decreased by $22 \%$ in the first year (vs 36\% in non-black/non-AA patients), then remained stable and above lower limit of normal in most patients.

Conclusions: Relapse rates remained low in black/AA patients, consistent with non-black/ non-AA patients. The safety profile of DMF in black/AA patients was consistent with that in the non-black/non-AA ESTEEM population, 
although lymphocyte decrease was less pronounced in black/AA patients.

Keywords: Dimethyl fumarate; Multiple sclerosis; Safety and effectiveness

\section{Key Summary Points}

\section{Why carry out this study?}

The oral multiple sclerosis (MS) therapy delayed-release dimethyl fumarate (DMF) has demonstrated clinically meaningful, sustained efficacy and a favorable benefit-risk profile in patients with relapsing forms of MS.

ESTEEM is an ongoing, 5-year, multinational, prospective study evaluating long-term safety and effectiveness of DMF in patients with MS.

Black or African American (AA) patients with MS are reported to exhibit greater disease severity compared with non-black/ non-AA patients.

MS clinical trials have included low numbers of non-white patients. This interim analysis from ESTEEM evaluated real-world safety and effectiveness of DMF in the largest subgroup of DMF-treated black or African American (black/AA) patients studied to date.

\section{What was learned from the study?}

The safety profile of DMF in black/AA patients was consistent with that in the non-black/non-AA ESTEEM population, although lymphocyte decrease was less pronounced in black/AA patients.

Relapse rates remained low in black/AA patients, consistent with non-black/nonAA patients.

Overall, these analyses demonstrate the real-world treatment benefit of DMF in black/AA patients, consistent with findings in the overall ESTEEM population.

\section{INTRODUCTION}

Multiple sclerosis (MS) has not been commonly studied in minority populations, and the very low level of participation by non-white patients is a well-recognized limitation of MS clinical trials [1]. Data describing the effects of diseasemodifying therapies in patients from minority groups treated in the real-world setting are similarly limited. As a result, MS is not well characterized in patient groups such as black or African American (AA) populations, leading to a lack of clinical information on evidence-based approaches to the most effective treatment [2]. Recently, there has been more focus on studying MS in minority populations, but these patient groups continue to be underrepresented in MS clinical trials. Also, among the studies that have been published, there was variation in the definitions of racial and ethnic subgroups.

Dimethyl fumarate (DMF) and diroximel fumarate are oral disease-modifying therapies approved for treating relapsing forms of MS. DMF and diroximel fumarate undergo esterase cleavage in the gut to monomethyl fumarate, a pharmacologically active metabolite $[3,4]$. As of January 31,2020 , more than 445,000 patients have been treated with DMF worldwide, representing more than 875,000 patient-years of exposure. Of these, 6335 patients $(14,241$ patient-years) were from clinical trials.

DMF has demonstrated significant, clinically meaningful, sustained efficacy and a favorable benefit-risk profile in patients with relapsing-remitting multiple sclerosis in the pivotal phase 3 studies DEFINE and CONFIRM, the ongoing open-label ENDORSE extension study, and real-world studies [5-7]. Although data from DEFINE and CONFIRM demonstrated a numerical reduction in relapses and disability progression in black patients, the sample size $(n=29)$ was too small to draw definitive conclusions regarding the effects of DMF in this subgroup [8]. A previous retrospective chart review demonstrated that DMF had similar effectiveness in AA $(n=69)$ and Hispanic American patients $(n=52)$ compared with white patients [9]. 
Table 1 Patient baseline characteristics

\begin{tabular}{|c|c|c|}
\hline$n(\%)$, unless otherwise specified & $\begin{array}{l}\text { Non-black/Non-AA } \\
\text { patients } \\
n=4897\end{array}$ & $\begin{array}{l}\text { Black/AA } \\
\text { patients } \\
n=187\end{array}$ \\
\hline Mean (SD) age, years & $40(11)$ & $43(11)$ \\
\hline \multicolumn{3}{|l|}{ Age category, years } \\
\hline$<40$ & $2498(51)$ & $73(39)$ \\
\hline$\geq 40$ & $2398(49)$ & $114(61)$ \\
\hline Sex, F & $3603(74)$ & $155(83)$ \\
\hline \multicolumn{3}{|l|}{ Region } \\
\hline USA, including Puerto Rico & $1216(25)$ & $181(97)$ \\
\hline Western Europe, Canada, New Zealand, and Australia & $2806(57)$ & $6(3)$ \\
\hline Eastern Europe plus India, Mexico, and Argentina & $875(18)$ & $0(0)$ \\
\hline Median (range) age at MS diagnosis, years & $34(9-83)$ & $36(15-64)$ \\
\hline $\begin{array}{l}\text { Median (range) duration between most recent relapse and enrollment, } \\
\text { months }\end{array}$ & $5.8(0-410)$ & $6.2(0-182)$ \\
\hline Median (range) number of relapses within past 12 months & $1(0-6)$ & $1(0-4)$ \\
\hline Mean (SD) DMF treatment duration, months & $21.5(16.2)$ & $21.2(17.0)$ \\
\hline Patients with minimum 1-year follow-up & $3124(64)$ & $114(61)$ \\
\hline Patients with minimum 2-year follow-up & $1877(38)$ & $70(37)$ \\
\hline Median (range) duration in ESTEEM, months & $24.4(0-63)$ & $23.2(2-60)$ \\
\hline Mean (SD) EDSS score ${ }^{b}$ & $2.10(1.48)$ & $3.17(2.29)$ \\
\hline Prior MS treatment & $3109(64)$ & $139(74)$ \\
\hline Glatiramer acetate & $1209(39)$ & $76(55)$ \\
\hline Intramuscular IFN $\beta$-1a & $932(30)$ & $54(39)$ \\
\hline Subcutaneous IFN $\beta-1 \mathrm{a}$ & $1488(48)$ & $56(40)$ \\
\hline IFN $\beta-1 b$ & $194(6)$ & $1(1)$ \\
\hline Fingolimod & $279(9)$ & $14(10)$ \\
\hline Teriflunomide & $213(7)$ & $8(6)$ \\
\hline Tobacco use & 2738 & 133 \\
\hline Current user & $705(26)$ & $21(16)$ \\
\hline Non-tobacco user & $2033(74)$ & $112(84)$ \\
\hline
\end{tabular}

AA African American, EDSS Expanded Disability Status Scale, IFN interferon, $M S$ multiple sclerosis

a Age $=$ (year diagnosed or year of first MS symptom - year of birth +1 )

b For patients with available data at enrollment from EDSS assessment. Non-black/non-AA patients, $n=2797$; black/AA patients, $n=24$ 
ESTEEM (ClinicalTrials.gov identifier NCT02047097) is an ongoing, 5-year, multinational, prospective, noninterventional, phase IV study evaluating the long-term safety and effectiveness of DMF in patients treated in realworld clinical practice. Results will include data from 5000 patients. In this interim analysis, we aimed to evaluate the safety and effectiveness of DMF on MS disease activity in routine clinical practice in non-black/non-AA patients, black/ AA patients, and a cohort of black/AA patients who switched from another therapy to DMF (received prior interferon [IFN]/glatiramer acetate $[\mathrm{GA}]$ at any time following MS diagnosis).

\section{METHODS}

\section{Study Design and Patients}

This interim analysis of the ESTEEM study (data cutoff, April 3, 2019) included patients with MS who had been newly prescribed DMF in routine practice at 394 sites globally. Race was self-reported and one of the given options for selfidentification was "Black or African American". Patients who did not identify as black or African American were recorded as non-black/non-AA. Patients under the age of 12 are excluded from the ESTEEM study, as $1 \%$ of the MS population is aged 11 years or younger [10]. Patients aged 12 to less than 18 years are also excluded where enrollment of pediatric patients is prohibited by local regulations. The primary objective of the ESTEEM study was to determine the incidence, type, and pattern of serious adverse events (SAEs), including serious infections, and of adverse events (AEs) leading to treatment discontinuation, while the secondary objective was to assess the effectiveness of DMF on MS clinical relapse activity. Magnetic resonance imaging data that would enable the characterization of disease severity at baseline were unavailable. The effectiveness of DMF on MS

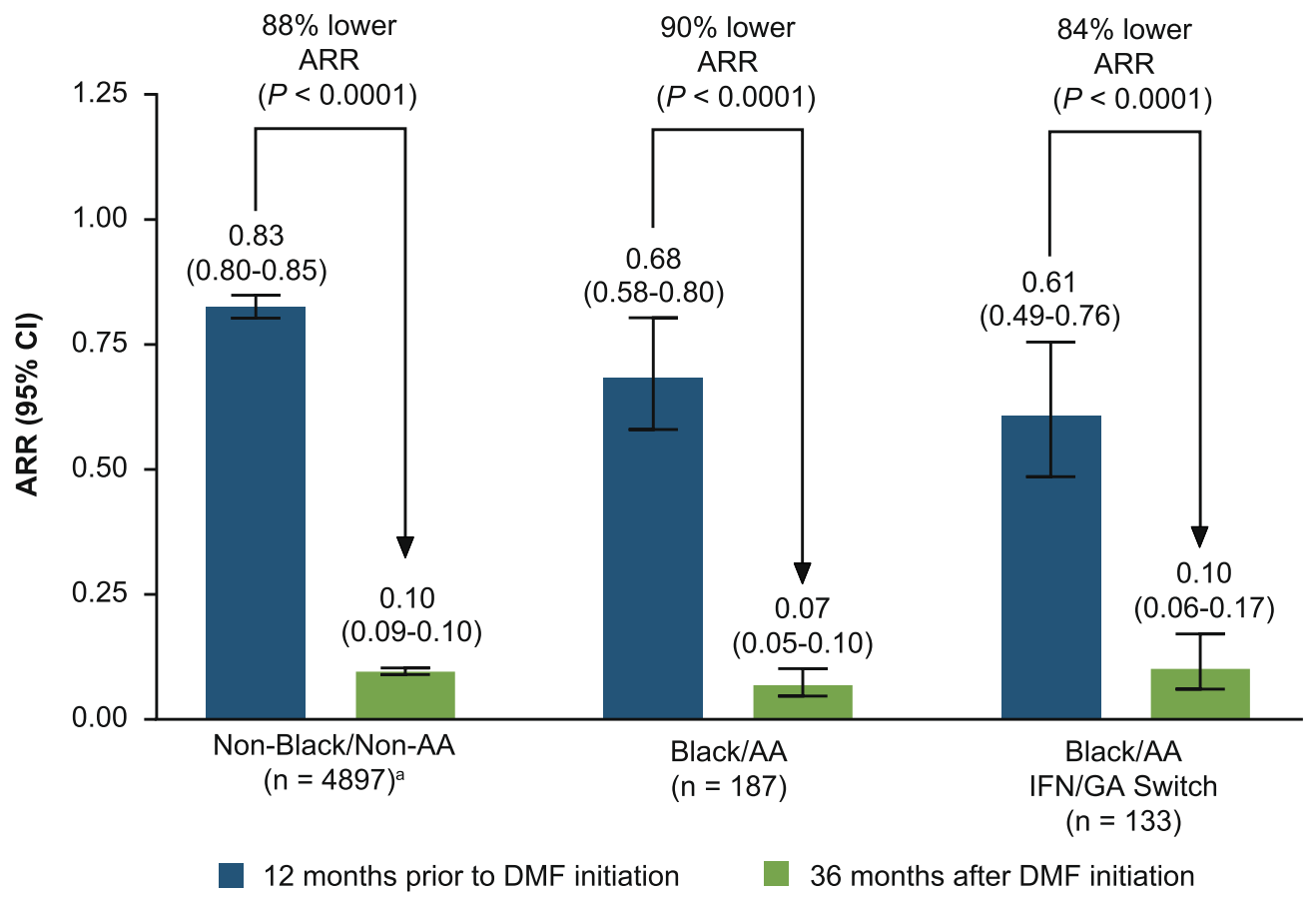

Fig. 1 ARR in the 12 months before and 36 months after DMF initiation for the non-black/non-AA population, the black/AA cohort, and the black/AA IFN/GA switch subgroup. $A A$ African American, $A R R$ annualized relapse rate, $D M F$ dimethyl fumarate. $G A$ glatiramer acetate, $I F N$ interferon. ${ }^{a}$ Patients with missing relapse-rate data 12 months before DMF initiation were excluded from the ARR analysis. ARRs were obtained by fitting a repeated-measure negative binomial model 
Table 2 Proportion of relapse-free patients in the 36 months after DMF initiation

\begin{tabular}{llll}
\hline & $\begin{array}{l}\text { Non-black/Non-AA } \\
\text { patients } \\
\boldsymbol{n}=\mathbf{4 8 9 7}\end{array}$ & $\begin{array}{l}\text { Black/AA } \\
\text { patients } \\
\boldsymbol{n}=\mathbf{1 8 7}\end{array}$ & $\begin{array}{l}\text { Black/AA IFN/GA switch } \\
\text { patients } \\
\boldsymbol{n}=\mathbf{1 3 3}\end{array}$ \\
\hline $\begin{array}{l}\text { Proportion of patients without MS } \\
\text { relapse }\end{array}$ & 80 & 82 & 79 \\
\hline
\end{tabular}

Values are given as percentage

$A A$ African American, $A R R$ annualized relapse rate, $D M F$ dimethyl fumarate, $G A$ glatiramer acetate, $I F N$ interferon, $M S$ multiple sclerosis

a Kaplan-Meier analyses

Table 3 Most common AEs leading to DMF discontinuation in the overall ESTEEM non-black/non-AA and black/AA patient population

\begin{tabular}{lll}
\hline Category, $\mathbf{n}(\%)^{\mathbf{a}}$ & $\begin{array}{l}\text { Non-black/Non-AA patients } \\
\boldsymbol{n}=\mathbf{4 8 9 7}\end{array}$ & $\begin{array}{l}\text { Black/AA patients } \\
\boldsymbol{n}=\mathbf{1 8 7}\end{array}$ \\
\hline Any AE leading to treatment discontinuation & $930(19)$ & $35(19)$ \\
GI disorders & $382(8)$ & $13(7)$ \\
Diarrhea & $112(2)$ & $6(3)$ \\
Nausea & $103(2)$ & $4(2)$ \\
Abdominal pain & $68(1)$ & $1(<1)$ \\
Vomiting & $60(1)$ & $4(2)$ \\
Skin and subcutaneous tissue disorders & $106(2)$ & $4(2)$ \\
Rash & $20(<1)$ & - \\
Rash, generalized & $7(<1)$ & - \\
Infections & $33(<1)$ & - \\
Investigations & $89(2)$ & $6(3)$ \\
Lymphocyte count decreased & $50(1)$ & $4(2)$ \\
Nervous system disorders & $96(2)$ & $7(4)$ \\
Blood and lymphatic system disorders & $141(3)$ & $4(2)$ \\
General disorders and administration site conditions & $67(1)$ & $2(1)$ \\
\hline
\end{tabular}

Values are given as number (percentage)

$A A$ African American, $A E$ adverse event, $D M F$ dimethyl fumarate, GI gastrointestinal

a AEs occurring in $>1$ patient in the black/AA subgroup. AEs were coded using the Medical Dictionary for Regulatory Activities (MedDRA) System Organ Class (SOC) and Preferred Term (PT). Percentages were calculated on the basis of total number of patients in each group. A patient was counted only once in each MedDRA SOC or PT 
disease activity was evaluated by assessment of annualized relapse rate (ARR), the proportion of patients with a relapse, and the distribution of the number of relapses. The incidence of treatment discontinuation was also assessed.

The study was conducted in accordance with relevant US federal regulations, the Declaration of Helsinki, and the International Council on Harmonisation Guideline for Good Clinical Practice. Approvals were granted by relevant institutional ethics committees for study protocol and amendments, and written assent and consent forms were obtained from each patient and his or her parent or legal guardian. ESTEEM was registered at ClinicalTrials.gov (NCT02047097).

\section{Statistical Analysis}

ARRs were obtained by fitting a repeated-measure negative binomial model (ARR was defined as the total number of relapses during the study for all patients divided by the total number of patient-years in the study adjusted for appropriate prognostic factors, and time-to-event endpoints analyzed using Kaplan-Meier estimates). Descriptive summary statistics were calculated.

\section{RESULTS}

\section{Study Population}

In total, 4897 non-black/non-AA patients, 187 black/AA patients, and 133 black/AA patients who switched to DMF from IFN/GA, received at least one dose of DMF, and were included in this analysis. The mean (SD) age of the black/AA subgroup was 43 (11) years, and $83 \%$ of these patients were female. No patients in the black/ AA patient group were less than 18 years of age (non-black/non-AA population, $n=10$; black/ AA population, $n=0$ [minimum age, 20 years]). Baseline characteristics for the black/AA and non-black/non-AA patient populations were generally similar (Table 1), although the majority of black/AA patients (97\%) were from the USA, and the percentage of patients over 40 years of age was significantly greater in the black/AA cohort $(61 \%$; 114/187) than in the non-black/non-AA population (49\%; 2398/4897; $P<0.0001$ ).

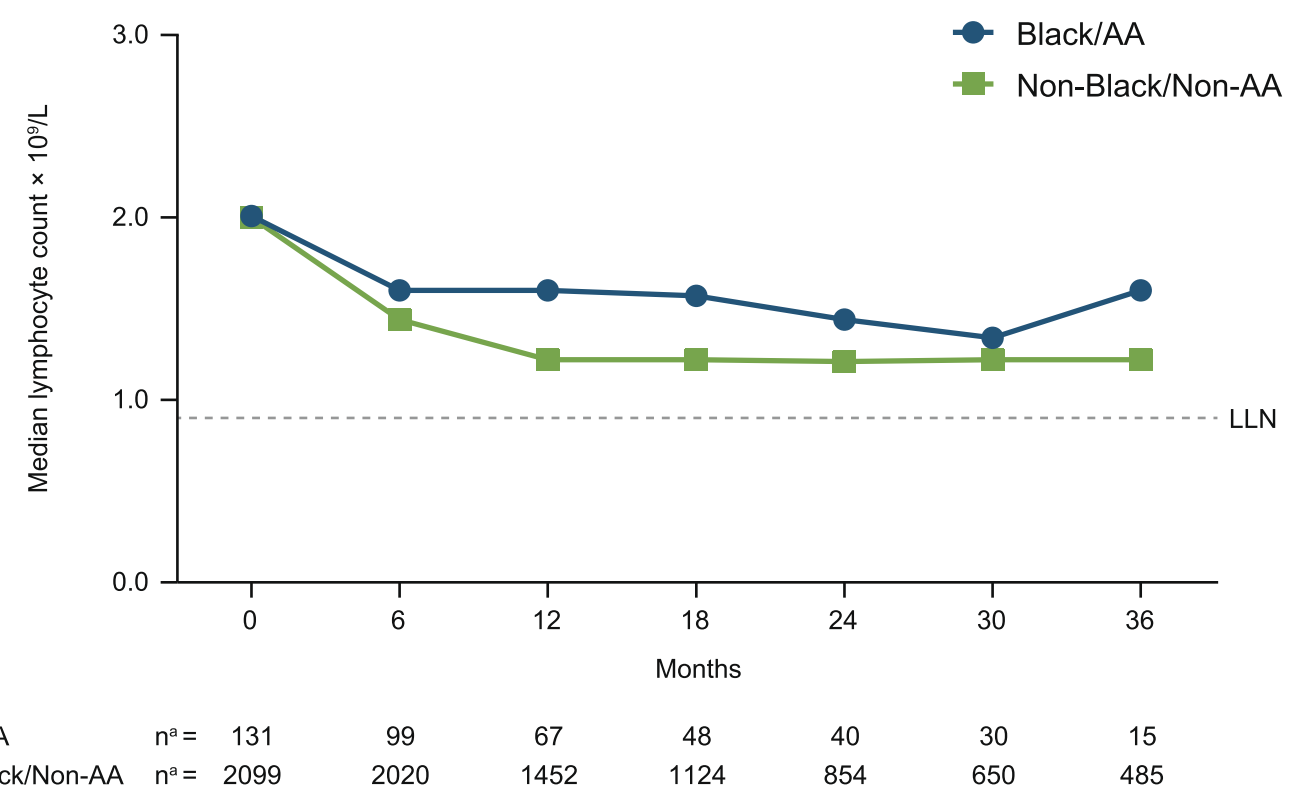

Fig. 2 Median absolute lymphocyte count over time in ${ }^{a} n=$ number of patients with available data from absolute non-black/non-AA and black/AA patients. $A A$ African American, $L L N$ lower limit of normal $\left(0.91 \times 10^{9} / \mathrm{L}\right)$. 


\section{ARR and Relapses}

The mean (SD) duration between most recent relapse and enrollment was 21.1 (36.30) months for the non-black/non-AA population and 22.8 (35.96) months for the black/AA population. The mean (SD) number of relapses in the prior 3 years was 1.4 (1.32) for the non-black/non-AA population and 1.2 (1.86) for the black/AA population. Among black/AA patients, ARR 12 months before DMF initiation was 0.68 (95\% CI $0.58-0.80$ ), and after 36 months of DMF treatment it was 0.07 (95\% CI 0.05-0.10; $P<0.0001$; Fig. 1). Similarly, ARR after 36 months of DMF in the black/AA IFN/GA switch subgroup was 0.10 (95\% CI 0.06-0.17), compared with ARR 12 months before DMF initiation of $0.61 \quad(95 \%$ CI $0.49-0.76$; $P<0.0001)$. The estimated proportion of patients without a relapse at 36 months ranged from $79 \%$ to $82 \%$ in the non-black/non-AA, black/AA, and black/AA IFN/GA switch subgroups (Table 2).

\section{Safety and Discontinuations}

A total of 930 (19\%) non-black/non-AA patients and 35 (19\%) black/AA patients reported any AE leading to treatment discontinuation (Table 3), with gastrointestinal (GI) disorders being the most commonly cited (non-black/non-AA, $n=382 \quad[8 \%] ;$ black/AA, $n=13 ; \quad[7 \%])$. The majority of these GI-related discontinuations occurred within the first month of DMF treatment (non-black/non-AA, $n=270 / 382$ [71\%]; black/AA, $n=9 / 13$ [69\%]). SAEs were reported in $237 / 4897(5 \%)$ non-black/non-AA patients and 8/187 (4\%) black/AA patients.

\section{Absolute Lymphocyte Counts}

In black/AA patients with available lymphocyte data $(n=67)$, median absolute lymphocyte count (ALC) decreased by $22 \%$ from baseline $\left(2.01 \times 10^{9} / \mathrm{L}\right)$ to month $12\left(1.60 \times 10^{9} / \mathrm{L}\right)$ and then remained stable and above the lower limit of normal $\left(0.91 \times 10^{9} / \mathrm{L}\right)$ in the majority of patients (Fig. 2). There was an increase in median absolute lymphocyte count from month 30 to month 36 in the black/AA cohort (Fig. 2); this may be attributable to the small sample size $(n=15)$ at year 3. In non-black/non-AA patients with available lymphocyte data $(n=2344)$, median ALC decreased by $36 \%$ from baseline $\left(1.97 \times 10^{9} / \mathrm{L}\right)$ to month $12\left(1.21 \times 10^{9} / \mathrm{L}\right)$ and then remained stable.

\section{DISCUSSION}

Here we report on the safety and effectiveness of DMF in black/AA patients versus non-black/ non-AA patients. Compared with the 12 months before DMF initiation, ARR was significantly lower in the 36 months after DMF initiation in the overall black/AA patient cohort, and in those black/AA patients who switched from prior IFN/GA to DMF. Importantly, these results are consistent with ARRs reported in the non-black/non-AA population. No unexpected safety findings were observed in the black/AA population, with $\mathrm{AE}$ and $\mathrm{SAE}$ profiles similar to those seen in non-black/nonAA patients.

In this study, a median $22 \%$ reduction in ALC was observed over the first year of DMF treatment in black/AA patients, which remained stable thereafter. For comparison, the decrease seen in non-black/non-AA patients was $36 \%$. Other authors have also noted a less pronounced decrease in ALC from baseline and lower incidence of lymphopenia in black/AA patients versus white patients $[9,11]$; the lower drop in lymphocytes observed in this study supports these findings. Another notable observation from our study related to ALC: at 36 months after DMF initiation there was a marked increase in ALC, which brought the median ALC higher than the baseline value; we attribute this increase to the small sample size at 36 months $(n=15)$. Further characterization of the long-term lymphocyte dynamics will be possible upon study completion.

The observational nature of this study is one of its strengths, as it enables understanding of safety and effectiveness in routine clinical care; however, the voluntary design of the study introduces risk of selection bias. Although baseline magnetic resonance imaging data were 
unavailable, the relapse history of the black/AA population indicates that these patients, as a group, did have active disease (ARR 0.68) within the year prior to DMF initiation, as most patients with MS do prior to starting therapy.

The number of black/AA patients included in this study is representative of the low levels of participation by black/AA patients in clinical trials overall. Despite only $4 \%$ of patients in the ESTEEM study, being of black/AA descent, this remains the largest black/AA MS research cohort to date in a prospective MS therapeutic study. There is a need for clinicians to discuss and encourage clinical trial participation with patients, especially those from minority racial populations, to better understand potential differences in MS disease prognosis and the degree to which race may impact response to therapy.

Although clinically important differences in the course of MS may exist among patients of different ethnic groups, previous studies are unclear with regard to whether there is also a variable response to therapies. Previous literature has suggested that patients of AA descent tend to have more frequent relapses, worse postrelapse recoveries, faster transition from relapsing-remitting to secondary-progressive MS, and more rapid disability progression compared with white Americans [12-15]. There is only one study in the literature that reports a poorer response to IFN $\beta$ therapy $(n=36)$ in AA patients than in a white American population [16]. There is also a study in teriflunomide-treated patients that found similar levels of treatment satisfaction in both AA and non-AA patients; however, there were higher relapse rates in AA patients ( 0.35 over 1 year in AA patients versus 0.16 over 1 year in non-AA patients) [17]. This less-than-optimal response in AA patients may be due to a potentially more severe course of MS disease activity and/or reduced efficacy response to IFN $\beta$ therapy or teriflunomide therapy in AA patients [18, 19]. However, the small sample size may have also contributed to a sampling error. Conversely, more recent subgroup analyses from an observational study of the safety and effectiveness of pegylated IFN $\beta-1 \mathrm{a}$ in a small cohort $(n=38)$ of AA patients demonstrated low relapse rates; however, the sample size was small and, similarly to the present study, these patients were selected for IFN therapy and were not randomized [20]. Natalizumab has also been shown to significantly lower both relapse rate and the accumulation of brain lesions in patients of African descent with relapsing-remitting multiple sclerosis $(n=49)$ [21]. These subanalyses are once again limited by the small numbers of participants, which affect the generalizability of the results. A further consideration is that multiple factors, including socioeconomic status and access to care (social determinants of health), therapy adherence patterns, and impact of comorbidities in different patient groups, may have a significant impact upon MS disease course outcomes. However, a study that examined adherence, persistence, and discontinuation in African American patients with MS treated with fingolimod or GA found medication compliance to be similar to that of the overall population of patients with MS in other studies [22].

Another limitation of this study is that most of the patients in this subgroup were from the USA and therefore may not be representative of a global MS population. Stratified analyses based on race are not available for patients from Europe because of differences in data collection and data privacy regulations at those study sites. Other studies may define racial and ethnic subgroups differently and the subgroup definitions have inconsistent nomenclature.

An interesting observation in the current study was that enrolled black/AA patients were significantly older than patients in the nonblack/non-AA cohort, which may suggest that black/AA patients are diagnosed later or are being managed differently. In conclusion, these safety and effectiveness analyses hitherto demonstrate the real-world treatment benefit of DMF in black/AA patients, consistent with findings in the overall ESTEEM population and in a subgroup analysis of Hispanic American patients [23]. The current study analyzed DMF outcomes in the largest black/AA cohort to date and reports outcomes over the longest followup duration compared with previous studies. As the ESTEEM study is still ongoing, the final 5-year ESTEEM study results may provide 
further insights into the safety and effectiveness of DMF in different ethnic groups.

\section{ACKNOWLEDGEMENTS}

Funding. This study was sponsored by Biogen (Cambridge, MA, USA). Including the Rapid Service Fee.

Medical Writing and/or Editorial Assistance. Biogen provided funding for medical writing support in the development of this paper; Katherine Ayling-Rouse, MSc, and Chris Traylen, PhD, from Excel Scientific Solutions wrote the first draft of the manuscript based on input from authors, and Adrienne Trout, BSc, from Excel Scientific Solutions copyedited and styled the manuscript per journal requirements. Biogen reviewed and provided feedback on the paper to the authors. The authors had full editorial control of the paper and provided their final approval of all content.

Authorship. All named authors meet the International Committee of Medical Journal Editors (ICMJE) criteria for authorship for this article, take responsibility for the integrity of the work as a whole, and have given their approval for this version to be published.

Disclosures. Dr. Williams has received advisory/consulting fees from Biogen, Celgene, EMD Serono, Genentech, Sanofi Genzyme, and Teva Neuroscience, and speaker fees from Biogen, Genentech, Sanofi Genzyme, and Teva. Dr. Amezcua has received advisory/consulting fees from Novartis and Genzyme, and research support from Biogen and MedDay. Dr. Okai has received advisory/consulting fees from Biogen, Celgene, EMD Serono, Genentech, Genzyme, and Novartis; research support from Biogen, Novartis, Sanofi Genzyme, and TG Therapeutics; and speaker fees from Biogen, Genentech, Novartis, Sanofi Genzyme, and Teva. Dr. Okuda has received advisory/consulting fees from Biogen, Celgene, EMD Serono, Genentech, Genzyme, and Novartis, and research support from Biogen and EMD Serono. Dr. Cohan has received advisory/consulting fees from Biogen, Celgene, Novartis, Pear Therapeutics, Roche Genentech, Sage Therapeutics, and Sanofi Genzyme; research support from AbbVie, Adamas, Alithios, Biogen, EMD Serono, MedDay, Novartis, Roche Genentech, and Sanofi Genzyme; and speaker fees from Biogen, Roche Genentech, and Sanofi Genzyme. Drs. Su, Mendoza, Lewin, and Jones are employees of and hold stock/stock options in Biogen. Dr Parks is now employed at Blueprint Medicines, Cambridge, MA, USA.

Compliance with Ethics Guidelines. The study was conducted in accordance with relevant US federal regulations, the Declaration of Helsinki, and the International Council on Harmonisation Guideline for Good Clinical Practice. Approvals were granted by relevant institutional ethics committees for study protocol and amendments, and written assent and consent forms were obtained from each patient and his or her parent or legal guardian. ESTEEM was registered at ClinicalTrials.gov (NCT02047097).

Data Availability. All data generated or analyzed during this interim subgroup analysis are included in this published article/as supplementary information files.

Open Access. This article is licensed under a Creative Commons Attribution-NonCommercial 4.0 International License, which permits any non-commercial use, sharing, adaptation, distribution and reproduction in any medium or format, as long as you give appropriate credit to the original author(s) and the source, provide a link to the Creative Commons licence, and indicate if changes were made. The images or other third party material in this article are included in the article's Creative Commons licence, unless indicated otherwise in a credit line to the material. If material is not included in the article's Creative Commons licence and your intended use is not permitted by statutory regulation or exceeds the permitted use, you will need to obtain permission directly from the copyright holder. To view a copy of this licence, 
visit http://creativecommons.org/licenses/bync/4.0/.

\section{REFERENCES}

1. MS Minority Research Engagement Partnership Network. Summary of barriers to research participation for minority individuals with MS 2017. https://www.pcori.org/sites/default/files/MS-Minori ty-Research-Engagement-Partnership-Barriers-to-Par ticipation.pdf. Accessed 14 Oct, 2019.

2. Khan O, Williams MJ, Amezcua L, Javed A, Larsen KE, Smrtka JM. Multiple sclerosis in US minority populations: clinical practice insights. Neurol Clin Pract. 2015;5(2):132-42.

3. TECFIDERA. TECFIDERA ${ }^{\circledR}$ (dimethyl fumarate) delayed-release capsules, for oral use. Highlights of prescribing information, Cambridge, MA, USA: Biogen; 2017. https://www.tecfidera.com/content/ dam/commercial/tecfidera/pat/en_us/pdf/full-presc ribing-info.pdf. Accessed 15 Oct, 2018.

4. US Food and Drug Administration. VUMERITY ${ }^{\mathrm{TM}}$ (diroximel fumarate) delayed-release capsules, for oral use. Highlights of prescribing information Cambridge, MA, USA: Biogen; 2019. https://www. accessdata.fda.gov/drugsatfda_docs/label/2019/211 855s000lbl.pdf. Accessed 10 Sep, 2019.

5. Fox RJ, Miller DH, Phillips JT, et al. Placebo-controlled phase 3 study of oral BG-12 or glatiramer in multiple sclerosis. N Engl J Med. 2012;367(12): 1087-97.

6. Gold R, Arnold DL, Bar-Or A, et al. Long-term effects of delayed-release dimethyl fumarate in multiple sclerosis: interim analysis of ENDORSE, a randomized extension study. Mult Scler. 2017;23(2):253-65.

7. Gold R, Kappos L, Arnold DL, et al. Placebo-controlled phase 3 study of oral BG-12 for relapsing multiple sclerosis. N Engl J Med. 2012;367(12): 1098-107.

8. Fox RJ, Gold R, Phillips JT, Okwuokenye M, Zhang A, Marantz JL. Efficacy and tolerability of delayedrelease dimethyl fumarate in black, Hispanic, and Asian patients with relapsing-remitting multiple sclerosis: post hoc integrated analysis of DEFINE and CONFIRM. Neurol Ther. 2017;6(2):175-87.

9. Zhovtis Ryerson L, Green R, Confident G, et al. Efficacy and tolerability of dimethyl fumarate in White-, African- and Hispanic- Americans with multiple sclerosis. Ther Adv Neurol Disord. 2016;9(6):454-61.

10. Gadoth N. Multiple sclerosis in children. Brain Dev. 2003;25(4):229-32.

11. Morales FS, Koralnik IJ, Gautam S, Samaan S, Sloane JA. Risk factors for lymphopenia in patients with relapsing-remitting multiple sclerosis treated with dimethyl fumarate. J Neurol. 2020;267(1):125-31.

12. Cree BA, Khan O, Bourdette D, et al. Clinical characteristics of African Americans vs Caucasian Americans with multiple sclerosis. Neurology. 2004;63(11):2039-45.

13. Kister I, Chamot E, Bacon JH, et al. Rapid disease course in African Americans with multiple sclerosis. Neurology. 2010;75(3):217-23.

14. Naismith RT, Trinkaus K, Cross AH. Phenotype and prognosis in African-Americans with multiple sclerosis: a retrospective chart review. Mult Scler. 2006;12(6):775-81.

15. Marrie RA, Cutter G, Tyry T, Vollmer T, Campagnolo D. Does multiple sclerosis-associated disability differ between races? Neurology. 2006;66(8): 1235-40.

16. Cree BA, Al-Sabbagh A, Bennett R, Goodin D. Response to interferon beta-1a treatment in African American multiple sclerosis patients. Arch Neurol. 2005;62(11):1681-3.

17. Okai A, Khatri B, Edwards KR, et al. Outcomes in the African American Subpopulation From the Phase 4 Teri-PRO Study of Teriflunomide. In: Presented at Consortium of Multiple Sclerosis Centers 2017 Annual Meeting. 2017.

18. Cree BA, Reich DE, Khan O, et al. Modification of multiple sclerosis phenotypes by African ancestry at HLA. Arch Neurol. 2009;66(2):226-33.

19. Klineova S, Nicholas J, Walker A. Response to disease modifying therapies in African Americans with multiple sclerosis. Ethn Dis. 2012;22(2):221-5.

20. Naylor ML, Salvetti M, Mokliatchouk O, Everage N, Popescu C, Wray S. Plegridy observational program third interim analysis: safety and effectiveness of peginterferon beta-1a in a subgroup of African American patients. Int J MS Care. 2019;21(suppl 1): 45 .

21. Cree BA, Stuart WH, Tornatore CS, Jeffery DR, Pace $\mathrm{AL}$, Cha $\mathrm{CH}$. Efficacy of natalizumab therapy in patients of African descent with relapsing multiple sclerosis: analysis of AFFIRM and SENTINEL data. Arch Neurol. 2011;68(4):464-8. 
22. Williams MJ, Johnson K, Trenz HM, et al. Adherence, persistence, and discontinuation among Hispanic and African American patients with multiple sclerosis treated with fingolimod or glatiramer acetate. Curr Med Res Opin. 2018;34(1):107-15.
23. Chinea A, Amezcua L, Vargas W, et al. Real-world safety and effectiveness of dimethyl fumarate in Hispanic or Latino patients with multiple sclerosis: 3-year results from ESTEEM. Neurol Ther. 2020. https://doi.org/10.1007/s40120-020-00192-6. 